\title{
The National Center for X-Ray Tomography: Status Update
}

Gerry McDermott ${ }^{1,2}$, Rosanne Boudreau ${ }^{1,2}$, Jian-Hua Chen ${ }^{1,2}$, Axel Ekman ${ }^{1,2}$, Mark LeGros ${ }^{1,2}$, Tia Plautz $^{1,2}$ \& Carolyn A. Larabel1 ${ }^{1,2}$

${ }^{1 .}$ Department of Anatomy, University of California San Francisco, San Francisco, CA, USA

${ }^{2}$ Molecular Biophysics and Integrated Bioimaging, Lawrence Berkeley Lab, Berkeley, CA, USA

The National Center for X-ray Tomography (NCXT) develops new technologies for bio-imaging. In particular, the NCXT pioneered the development of soft x-ray tomography (SXT) as a method for imaging whole, hydrated cells, including eukaryotic cells. This presentation will describe the current status of this work, together our progress incorporating 'super-resolution' cryogenic SIM as a correlative partner for SXT.

Imaging is a fundamental component in most cell biology research. The NCXT has worked to address unmet imaging needs in this regard. In particular, we have developed methods for visualizing and quantifying cells and their sub-cellular organization.

Soft $\mathrm{x}$-ray tomography is a non-invasive, 3D imaging technique that visualizes and quantifies volumes, surfaces, interfaces, membranes, and organelle connectivity within an intact cell [1]. In a soft x-ray microscope the specimen is illuminated with photons with energies that lies within a spectral region known as the 'water window' $[2,3]$. In this regime, the illumination is absorbed more strongly by bio-molecules than by water, resulting in the generation of image contrast without the need for strains [4]. Water window light can penetrate intact, fully hydrated cells, including mammalian cells, and still produce images that have good signal-to-noise [5]. This is a marked advantage over electron microscopy, where most types of cell must be thinned - either by milling or sectioning - before they can be imaged [6].

All high resolution imaging methods have the potential to cause accumulated photon damage to the specimen, which in turn induces visible artifacts. Fixation, in most instances, mitigates this damage to undetectable levels [7]. Prior to being imaged by SXT, specimens are therefore cryo-preserved. As a result, biological samples can be imaged repeatedly - as is required in the acquisition of tomographic data -without signs of radiation damage [8]. Cryo-preserved specimens are regarded as being very close to 'native state', that is to say the internal organization is identical to that of a normal, functioning cell. In this case, high-resolution imaging makes perfect sense. SXT visualization the cell architecture relates directly with the observed in vivo function.

While SXT produces stunning, high-resolution views of cell features it can't directly image individual molecules $[9,10]$. Determining the location of particular molecules inside the cell is an essential step in many projects. Consequently, we have been developing cryogenic fluorescence microscopy methods that determine the position of tagged-molecules in cells imaged by SXT. Working with cryo-preserved cells means molecules localized by fluorescence will be locked in the same position when the cell is imaged by SXT. Our original 'proof of concept' cryogenic fluorescence micoscope was diffraction limited. In other words, it had a spatial resolution of many hundreds of $\mathrm{nm}$ [8, 11-13]. Since SXT images have a spatial resolution of 50nm or better, we designed and built a 'super-resolution' cryolight microscope. The new instrument is a cryogenic Structured Illumination Microscope (cryo-SIM) and is capable of localizing 
molecules at a spatial resolution equal (or even better) than SXT. This is the ideal in correlated imaging studies, since parity of resolution eliminates uncertainties interpretating two overlaid data sets.

SXT has evolved during the past decade and is now a mature imaging modality for cell biology. However, while the technique is mature in utility and community acceptance, SXT is still far from reaching a technological plateau. New nano-fabrication methods are producing soft x-ray microscope lenses with ever decreasing line-widths, allowing for even higher resolution imaging. Correlated imaging is also far from reaching its technological potential. In the near future, it is easy to imagine an individual cell being imaged/analyzed by more than two techniques. And finally, all imaging depends on access to high quality specimens. There is much potential in the development of new technologies for enhanced specimen isolation and preparation. In an idea future, it will be possible to rapidly select and correlatively image a particular cell from a large, heterogenous population of cells. The result will be an SXT reconstruction of the cell, decorated with the locations of a handful of different molecules. And, all of this will be done with minimal human input.

\section{References:}

[1] Larabell, C.A. and K.A. Nugent, Current Opinion in Structural Biology 20 (2010), p. 623.

[2] Parkinson, D.Y. et al, J Struct Biol 162 (2008), p. 380.

[3] Le Gros, M.A. et al, J Synchrotron Radiat 21 (2014), p. 1370.

[4] Parkinson, D.Y. et al, Methods Mol Biol 950 (2013), p. 457.

[5] Ekman, A.A. et al, Biol Cell 109 (2017), p. 24.

[6] Do, M. et al, Arch Biochem Biophys 581 (2015), p. 111.

[7] McDermott, G., M.A. Le Gros, and C.A. Larabell, Annu Rev Phys Chem 63 (2012), p. 225.

[8] Smith, E.A., et al., in "Correlated soft x-ray tomography and cryo-light microscopy, in Imaging Life: Biological Systems from Atoms to Tissues”, ed. G.C. Howard, W.E. Brown, and M. Auer. (Oxford University Press, USA).

[9] Cinquin, B.P. et al, J Cell Biochem 115 (2014), p. 209.

[10] Smith, E.A. et al, J Struct Biol 184 (2013), p. 12.

[11] Smith, E.A. et al, Biophysical Journal 106 (2014), p. 434a.

[12] Smith, E.A. et al, Biophys J 107 (2014), p. 1988.

[13] Le Gros, M.A. et al, J Microsc 235 (2009), p. 1. 